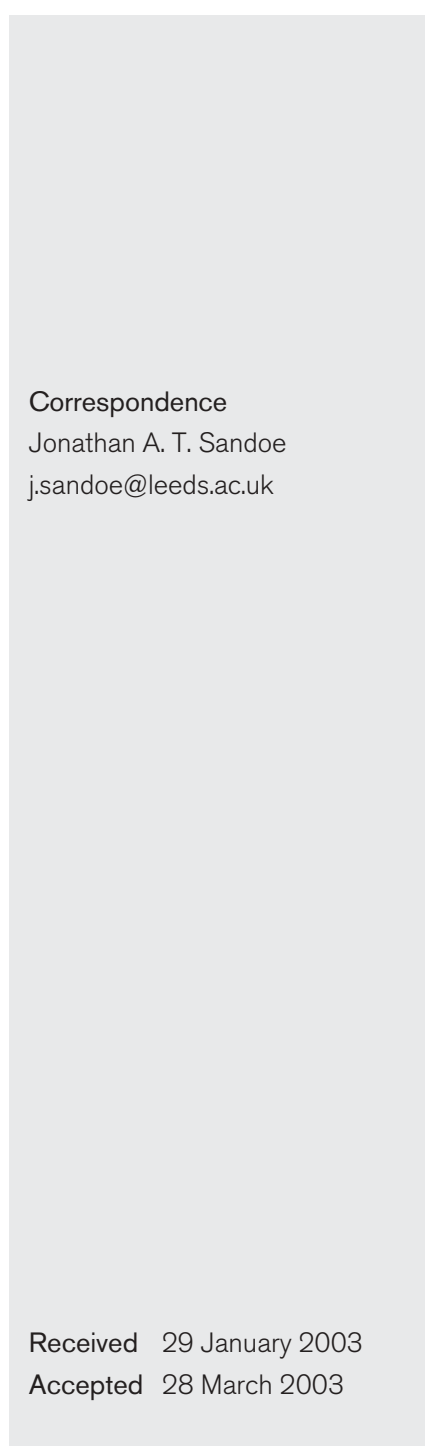

\title{
Correlation between enterococcal biofilm formation in vitro and medical-device-related infection potential in vivo
}

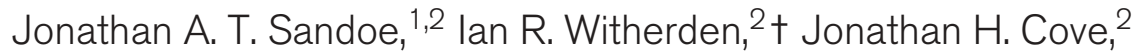 \\ John Heritage ${ }^{2}$ and Mark H. Wilcox ${ }^{1,2}$ \\ Department of Microbiology, The General Infirmary at Leeds ${ }^{1}$ and University of Leeds ${ }^{2}$, Leeds \\ LS1 3EX, UK
}

\begin{abstract}
Hospital-acquired infections caused by enterococci have increased dramatically since the 1970s. Many nosocomial enterococcal bloodstream infections are associated with medical devices such as central venous catheters. The ability to form biofilm on medical devices is a potential virulence trait that may allow enterococci to cause infections in the expanding population of patients managed with such devices. In this study, the hypothesis that increased ability to form biofilm in vitro is associated with medical-device-related infection in vivo was tested. A microplate assay was employed to assess biofilm-forming characteristics of enterococci in $0.9 \%(\mathrm{w} / \mathrm{v})$ sodium chloride, an oligotrophic environment, and $\mathrm{BHI}$, a nutrient-rich environment. Results were compared in isolates from different sources of infection. One hundred and nine enterococcal bloodstream isolates were assayed.

Biofilm formation on microplates was demonstrated by all Enterococcus faecalis isolates and 16/38 (42\%) Enterococcus faecium isolates. E. faecalis isolates produced significantly more biofilm than E. faecium isolates in both media $(P<0.0001$, Mann-Whitney $U$ test). E. faecalis isolates from intravascular-catheter-related bloodstream infections (CRBSIs) produced significantly more biofilm than non-CRBSI isolates $(P<0.0001)$, or isolates of uncertain clinical significance $(P<0.0001)$. Biofilm formed by $E$. faecium isolates was not significantly affected by culture medium and did not differ between isolates from the different clinical categories. In conclusion, there was significantly more biofilm formed by $E$. faecalis isolates causing CRBSI compared with isolates from other types of infection or from isolates of uncertain clinical significance. The ability of $E$. faecalis isolates to form biofilm in vitro appears to be a marker of a virulence trait that enhances the ability of isolates to cause CRBSI.
\end{abstract}

\section{INTRODUCTION}

Hospital-acquired infections caused by enterococci have increased dramatically since the 1970s. Enterococci account for approximately $7 \%$ of all bloodstream infections in Europe and $10 \%$ of bloodstream infections occurring on US intensive care units (Fluit et al., 2001; Fridkin \& Gaynes, 1999). Selection pressure from antibiotic use is a risk factor for enterococcal infection, but the observed increase in incidence predates the emergence of clinically important antimicrobial resistance in this genus (Toledo-Arana et al., 2001). In addition, clinical isolates of Enterococcus faecalis remain susceptible to the first- and second-line antimicrobial agents ampicillin and vancomycin in 85 and $95 \%$ of cases,

tPresent address: Academic Renal Unit, Division of Medicine, University of Bristol, UK.

Abbreviation: CRBSI, intravascular-catheter-related bloodstream infection. respectively (Reacher et al., 2000). Antibiotic use, therefore, is unlikely to be solely responsible for the emergence of enterococci in hospitals, and acquisition of new virulence traits may have played an important evolutionary role (Jett et al., 1994). The ability to form biofilm on medical devices is a putative virulence trait that may have enhanced the capacity of enterococci to cause infections. The proportion of enterococcal bacteraemias associated with central venous catheters has increased dramatically since the early 1980s (Malone et al., 1986; Patterson et al., 1995; Shlaes et al., 1981), reaching $35 \%$ in one UK study (Gray et al., 1994). Enterococcal intravascular-catheter-related bloodstream infection (CRBSI) causes significant morbidity and can be difficult to treat (Sandoe et al., 2002).

Once an intravascular catheter has become colonized with micro-organisms, invasion of the bloodstream can occur (Darouiche, 2001). In order to colonize catheters, a microorganism must have the capacity to form a biofilm on the 
device material. Clinical enterococcal strains have been shown to adhere to biomedical polymers in vitro, but the clinical relevance of this finding has not been determined (Joyanes et al., 1999). The aim of this study was to compare the biofilm-forming characteristics of bloodstream isolates in vitro and the type of infection caused in vivo to determine whether this trait may contribute to enterococcal virulence.

\section{METHODS}

Case identification. Searching the diagnostic microbiology laboratory database identified all patients with enterococcal bacteraemia in the General Infirmary at Leeds during 1998 and provided information regarding other microbiological investigations. Clinical information was obtained from the Department of Microbiology records collected during routine clinical liaison.

Clinical definitions. Enterococcal bacteraemia was defined as isolation of enterococci from a blood culture, with no implication of clinical significance. Enterococcal bloodstream infection was defined as isolation of enterococci from at least two blood culture sets, or isolation from at least one blood culture set and isolation of enterococci from a normally sterile site or clinical focus of infection. Episodes of enterococcal bloodstream infection were then categorized according to the focus of infection. CRBSI was defined as described previously (Sandoe et al., 2002). Validated criteria were used to define cases of endocarditis (Durack et al., 1994). Bloodstream infection occurring in the absence of enterococcal isolation from another site was categorized as being of unknown origin. When enterococci were isolated from at least one blood culture set mixed with other flora typically found in the gastrointestinal tract they were considered of gastrointestinal origin, unless another focus of infection was clinically apparent. Episodes of bacteraemia that did not satisfy any of the definitions above were considered of uncertain clinical significance, possibly representing skin contaminants or a transient bacteraemia.

Microbiological methods. Isolates were stored frozen at $-70{ }^{\circ} \mathrm{C}$ in nutrient broth No. 2 (Oxoid) containing 15\% glycerol (Fisher Scientific). Organisms were recovered from frozen stocks by plating out a $5 \mu$ l loop-full of frozen broth onto horse blood agar (HBA; Oxoid) and into $10 \mathrm{ml}$ brain heart infusion broth (BHI; Oxoid) and incubating at $37^{\circ} \mathrm{C}$ in air overnight. During the period of biofilm analysis, strains were subcultured to HBA plates every 4 weeks for a maximum of six times, before returning to frozen stocks. For the biofilm assay, isolates were picked from HBA plates and enriched in BHI broth for $24 \mathrm{~h}$ prior to performing the assay as described below. Enterococci were identified to genus and species level essentially as described previously (Facklam et al., 1999). Enterococci were distinguished from lactococci by the presence of Lancefield group D antigen, detected using the Streptococcal Grouping Kit (Oxoid). Pyrolydonyl arrylamidase activity was detected using PYR diagnostic tablets (A/S Rosco). Vancomycin susceptibility was omitted from the identification scheme because of the increasing prevalence of vancomycin-resistant enterococci.

Biofilm assays. Three colonies of test Enterococcus grown on HBA plates (that ranged from 1 to 30 days old) were inoculated into $10 \mathrm{ml}$ $\mathrm{BHI}$ broth and incubated without shaking, overnight, at $37^{\circ} \mathrm{C}$ in air. Cells were washed twice in sterile $0.9 \%$ (w/v) sodium chloride. Duplicate wells of sterile, polystyrene, 96-well, flat-bottomed tissue culture plates [Nalgene (Europe)] were each filled with $180 \mu \mathrm{l} \mathrm{BHI} \mathrm{or} \mathrm{0.9 \%} \mathrm{(w/}$ v) sodium chloride (saline), and $20 \mu \mathrm{l}$ of the washed cell suspension was added. Plates were incubated for $24 \mathrm{~h}$ in air supplemented with $5 \% \mathrm{CO}_{2}$ at $37^{\circ} \mathrm{C}$ for $24 \mathrm{~h}$. The contents of the wells were removed by inversion of the plates and gentle shaking. Microplate wells were then washed once by immersion of the entire plate in a plastic box containing approxi- mately $300 \mathrm{ml}$ sterile saline. Residual biofilm was initially fixed using $12 \%$ formaldehyde for $30 \mathrm{~min}$ but in later experiments microplates were simply left to air-dry overnight. Biofilms were then stained with filtered crystal violet (bioMérieux). The $\mathrm{OD}_{590}$ of each well was measured using a microplate reader (Spectromax 190; Molecular Devices). The mean optical density of blank wells plus three standard deviations was subtracted from individual readings for each experiment to give final corrected optical density results. Any optical density value greater than 0 after correction was considered positive. Experiments were repeated three times on different days, from which mean values were calculated. Assays were undertaken without prior knowledge of clinical categorization.

\section{RESULTS AND DISCUSSION}

\section{Focus of bloodstream infections}

One hundred and nine episodes of enterococcal bacteraemia occurred during 1998 in patients at Leeds General Infirmary. These comprised 65 enterococcal bloodstream infections and 44 episodes of uncertain clinical significance. Of the bloodstream infections, 22 were CRBSIs. The 43 non-CRBSIs included: 15 infections of unknown origin (35\%), four urinary tract infections $(9 \%), 17$ infections of gastrointestinal origin ( $40 \%$ ), five cases of endocarditis ( $12 \%$ ), one case of chorioamnionitis (2\%) and one soft-tissue infection ( $2 \%)$. The numbers of episodes in some types of infection were too small to allow individual analysis, so clinically significant non-CRBSIs were grouped together for subsequent comparisons. E. faecalis and E. faecium differed in the types of infection they caused; all of the CRBSIs were caused by $E$. faecalis and a far greater proportion of episodes of uncertain significance were caused by E. faecium (61\% vs $30 \% ; P=0 \cdot 004$, Fisher's exact test).

Prior to the emergence of enterococci as nosocomial pathogens, they were a recognized cause of endocarditis and urinary tract infection (Gilmore et al., 2002). These infections were considered to be endogenous, arising from the patient's own gastrointestinal flora. The pathogenesis of nosocomial enterococcal infection, however, appears to be different. Such infection follows colonization with nosocomial strains, which are presumed to possess virulence traits that set them apart from enterococci of the normal gastrointestinal flora (Gilmore et al., 2002). CRBSI is an important hospital-associated infection, accounting for $34 \%$ of all enterococcal bloodstream infections in this study, making it the commonest single source of invasive enterococcal infection. This finding is consistent with a previous study of enterococcal bacteraemia in the UK, in which intravascular catheters accounted for $35 \%$ of episodes (Gray et al., 1994). Although enterococci are known to cause urinary tract infections, only a small proportion $(6 \%)$ of the enterococcal bloodstream infections reported herein were from a confirmed urinary source, and none of these was associated with a urinary catheter. In common with other studies of enterococcal bacteraemia, in a large proportion $(23 \%)$ of episodes, the source of infection could not be determined (Garrison et al., 1982; Klimek et al., 1980; Maki \& Agger, 1988; Shlaes et al., 1981). 


\section{Biofilm formation}

One hundred and nine enterococcal bloodstream isolates, one from each episode of bacteraemia that occurred during 1998, were assayed for biofilm-forming ability. The enterococci comprised 70 E. faecalis, 38 E. faecium and 1 Enterococcus spp. Biofilm formation on microplates was demonstrated by all E. faecalis isolates and 16/38 (42\%) E. faecium isolates. Results of comparisons between the two predominant species are shown in Table 1. E. faecalis isolates produced significantly more biofilm than E. faecium isolates in both media $(P<0.0001$, Mann-Whitney U test). Because of marked differences in biofilm formation and infection type caused by the two predominant species, comparisons between type of infection and the ability to form biofilm were carried out separately for each species. E. faecalis CRBSI isolates produced significantly more biofilm than nonCRBSI isolates $(P<0 \cdot 0001$, Mann-Whitney $\mathrm{U})$, or isolates of uncertain clinical significance $(P<0.0001$, MannWhitney U) (Table 2). E. faecalis isolates of uncertain clinical significance did not differ significantly from non-CRBSI isolates in saline or BHI $(P<0 \cdot 05$, Mann-Whitney U). E. faecalis isolates produced higher optical density values in saline when compared with BHI $(P<0.0001$, Wilcoxon signed-rank test). Biofilm formed by E. faecium isolates was not significantly affected by culture medium and did not differ between isolates from the two clinical categories.

E. faecalis does not proliferate in an oligotrophic environment but has been shown to survive in tap water, without apparent changes to surface morphology within the first $24 \mathrm{~h}$ (Hartke et al., 1998). Overall, the biofilm formed by $E$. faecalis in saline was greater than that formed in BHI (see Table 2). Thus nutrient-starved E. faecalis can form biofilm on an inert surface and the stress response may actually enhance the biofilm formed in vitro by some strains. Although the ability to form biofilm on polystyrene microplates was demonstrated by all E. faecalis isolates, and may be an intrinsic feature of this species, those that caused CRBSI produced significantly more biofilm than the other groups. E. faecalis produced more biofilm than E. faecium, in accordance with previous investigations of enterococcal adhesion (Joyanes et al., 1999) and biofilm formation (Toledo-Arana et al., 2001).

Since the nature of the biomedical polymer does not affect enterococcal adherence significantly (Joyanes et al., 1999), in the present investigation biofilm formation on polystyrene microplates was used as a marker of biofilm formation on an unconditioned biomedical polymer. The finding that isolates from episodes of CRBSI produced more biofilm than isolates from non-device-related infections suggests that the in vitro model used in these experiments does have clinical relevance. An enhanced ability to form biofilm on biomedical polymers may increase the ability of enterococci to cause such infections and may therefore be an important virulence trait. Further support for this theory comes from the observation that E. faecium isolates were significantly less able to form biofilms and caused no episodes of CRBSI.

There are several difficulties in studying enterococcal virulence traits, and limitations of the present study, that warrant discussion. Firstly, no virulence traits have been identified that are present in all clinical enterococcal isolates. The putative virulence traits identified to date, such as production of haemolysin, aggregation substance and gelatinase, all have data supporting and dismissing their contribution to virulence. Traditionally, the search for enterococcal virulence factors has involved comparing clinical isolates with commensal faecal isolates from healthy individuals and demonstrating enrichment of the trait in clinical isolates (Murray, 1990). The choice of isolates for both test and control groups is particularly difficult, in part due to difficulty in attributing clinical significance to enterococci cultured from non-sterile sites (Murray, 1990). Furthermore, commensal faecal isolates can be expected to contain, as a subset, strains with enhanced virulence and the potential to cause infection. For this reason, blood culture isolates were chosen for the present study. Since enterococci can colonize the skin transiently, they can contaminate blood cultures and not every enterococcal bloodstream isolate is clinically relevant. To circumvent the problems of control group selection and clinical significance determination, strict definitions were applied to each episode of enterococcal bacteraemia and categorization according to focus of infection and likely clinical significance was undertaken. This approach enabled comparison between clinically significant isolates and those causing a transient bacteraemia of uncertain clinical significance or a 'pseudobacteraemia' (blood culture contaminant). The mechanisms behind biofilm formation are unknown. Although a role for enterococcal surface

Table $1 . \mathrm{OD}_{590}$ of $E$. faecalis and $E$. faecium biofilms after incubation in various culture media

\begin{tabular}{|c|c|c|c|c|c|c|c|}
\hline \multirow[t]{2}{*}{ Culture medium } & \multicolumn{3}{|c|}{ E. faecalis $(n=70)$} & \multicolumn{3}{|c|}{ E. faecium $(n=38)$} & \multirow[t]{2}{*}{$P$ value ${ }^{\star}$} \\
\hline & Mean & Median & Range & Mean & Median & Range & \\
\hline $\mathrm{NaCl}(0.9 \%, \mathrm{w} / \mathrm{v})$ & $0 \cdot 135$ & $0 \cdot 126$ & $0-0 \cdot 249$ & $0 \cdot 029$ & $0 \cdot 000$ & $0-0 \cdot 278$ & $<0.0001$ \\
\hline BHI & $0 \cdot 072$ & $0 \cdot 066$ & $0-0 \cdot 225$ & $0 \cdot 025$ & $0 \cdot 003$ & $0-0 \cdot 179$ & $<0.0001$ \\
\hline
\end{tabular}

${ }^{\star} P$ value shows results of the Mann-Whitney $\mathrm{U}$ test comparing E. faecium with E. faecalis. 
Table 2. Results of biofilm assays for isolates from different sources of enterococcal bloodstream infection

\begin{tabular}{|c|c|c|c|c|c|c|}
\hline \multirow[t]{3}{*}{ Type of infection $(n)$} & \multicolumn{6}{|c|}{$\mathrm{OD}_{590}$ after incubation in: } \\
\hline & \multicolumn{3}{|c|}{$\mathrm{NaCl}$} & \multicolumn{3}{|c|}{ BHI } \\
\hline & Mean & Median & Range & Mean & Median & Range \\
\hline \multicolumn{7}{|l|}{ E. faecium (38) } \\
\hline CRBSI $(0)$ & - & - & - & - & - & - \\
\hline Significant non-CRBSI (15) & $0 \cdot 027$ & $0 \cdot 000$ & $0-0 \cdot 163$ & $0 \cdot 023$ & $0 \cdot 012$ & $0-0 \cdot 103$ \\
\hline Uncertain clinical significance (23) & $0 \cdot 030$ & $0 \cdot 000$ & $0-0 \cdot 278$ & $0 \cdot 026$ & $0 \cdot 003$ & $0-0 \cdot 179$ \\
\hline \multicolumn{7}{|l|}{ E. faecalis (70) } \\
\hline $\operatorname{CRBSI}(22)^{\star}$ & $0 \cdot 174$ & $0 \cdot 182$ & $0 \cdot 075-0 \cdot 249$ & $0 \cdot 094$ & $0 \cdot 084$ & $0-0 \cdot 180$ \\
\hline Significant non-CRBSI (27) & $0 \cdot 108$ & $0 \cdot 102$ & $0 \cdot 025-0 \cdot 247$ & $0 \cdot 061$ & $0 \cdot 057$ & $0 \cdot 004-0 \cdot 140$ \\
\hline Uncertain clinical significance (21) & $0 \cdot 130$ & $0 \cdot 108$ & $0 \cdot 037-0 \cdot 246$ & $0 \cdot 065$ & $0 \cdot 062$ & $0 \cdot 008-0 \cdot 225$ \\
\hline
\end{tabular}

${ }^{\star}$ CRBSI isolates were significantly greater than non-CRBSI or isolates of uncertain significance in both media, $P<0 \cdot 0001$.

protein in biofilm formation has been proposed (ToledoArana et al., 2001), we could find no association between esp detection and biofilm-forming ability (data not shown).

In conclusion, E. faecalis was more likely to cause CRBSI than E. faecium and was also more likely to cause clinically significant bloodstream infection. E. faecalis isolates produced significantly more biofilm than E. faecium isolates. There was significantly more biofilm formed by E. faecalis isolates causing CRBSI compared with isolates from other types of infection or from isolates of uncertain clinical significance. The ability of enterococcal isolates to form biofilm in vitro appears to be a marker of a virulence trait that enhances the ability of isolates to cause CRBSI.

\section{REFERENCES}

Darouiche, R. O. (2001). Device-associated infections: a macroproblem that starts with microadherence. Clin Infect Dis 33, 1567-1572.

Durack, D. T., Lukes, A. S. \& Bright, D. K. (1994). New criteria for diagnosis of infective endocarditis: utilization of specific echocardiographic findings. Duke Endocarditis Service. Am J Med 96, 200-209.

Facklam, R. R., Sahm, D. F. \& Teixeira, L. M. (1999). Enterococcus. In Manual of Clinical Microbiology, pp. 297-305. Edited by P. R. Murray, E. J. Baron, M. A. Pfaller, F. C. Tenover \& R. H. Yolken. Washington, DC: American Society for Microbiology.

Fluit, A. C., Schmitz, F. J. \& Verhoef, J. (2001). Frequency of isolation of pathogens from bloodstream, nosocomial pneumonia, skin and soft tissue, and urinary tract infections occurring in European patients. Eur J Clin Microbiol Infect Dis 20, 188-191.

Fridkin, S. K. \& Gaynes, R. P. (1999). Antimicrobial resistance in intensive care units. Clin Chest Med 20, 303-316.

Garrison, R. N., Fry, D. E., Berberich, S. \& Polk, H. C., Jr (1982). Enterococcal bacteremia: clinical implications and determinants of death. Ann Surg 196, 43-47.

Gilmore, M. S., Coburn, P. S., Nallapareddy, S. R. \& Murray, B. E. (2002). Enterococcal virulence. In The Enterococci, pp. 301-354. Edited by M. S. Gilmore. Washington, DC: American Society for Microbiology.
Gray, J., Marsh, P. J., Stewart, D. \& Pedler, S. J. (1994). Enterococcal bacteraemia: a prospective study of 125 episodes. J Hosp Infect 27, 179-186.

Hartke, A., Giard, J. C., Laplace, J. M. \& Auffray, Y. (1998). Survival of Enterococcus faecalis in an oligotrophic microcosm: changes in morphology, development of general stress resistance, and analysis of protein synthesis. Appl Environ Microbiol 64, 4238-4245.

Jett, B. D., Huycke, M. M. \& Gilmore, M. S. (1994). Virulence of enterococci. Clin Microbiol Rev 7, 462-478.

Joyanes, P., Pascual, A., Martinez-Martinez, L., Hevia, A. \& Perea, E. J. (1999). In vitro adherence of Enterococcus faecalis and Enterococcus faecium to plastic biomaterials. Clin Microbiol Infect 5, 382-386.

Klimek, J. J., Ajemian, E., Gracewski, J., Klemas, B., Rios, I., Maderazo, E. \& Quintiliani, R. (1980). Enterococcal infections in a large community hospital, with emphasis on bacteremia. Am J Infect Control 8, 58-61.

Maki, D. G. \& Agger, W. A. (1988). Enterococcal bacteremia: clinical features, the risk of endocarditis, and management. Medicine (Baltimore) 67, 248-269.

Malone, D. A., Wagner, R. A., Myers, J. P. \& Watanakunakorn, C. (1986). Enterococcal bacteremia in two large community teaching hospitals. Am J Med 81, 601-606.

Murray, B. E. (1990). The life and times of the Enterococcus. Clin Microbiol Rev 3, 46-65.

Patterson, J. E., Sweeney, A. H., Simms, M., Carley, N., Mangi, R., Sabetta, J. \& Lyons, R. W. (1995). An analysis of 110 serious enterococcal infections: epidemiology, antibiotic susceptibility, and outcome. Medicine (Baltimore) 74, 191-200.

Reacher, M. H., Shah, A., Livermore, D. M. \& 8 other authors (2000). Bacteraemia and antibiotic resistance of its pathogens reported in England and Wales between 1990 and 1998: trend analysis. Br Med J320, 213-216.

Sandoe, J. A. T., Witherden, I. R., Au-Yeung, H.-K. C., Kite, P., Kerr, K. G. \& Wilcox, M. H. (2002). Enterococcal intravascular catheter-related bloodstream infection: management and outcome of 61 consecutive cases. J Antimicrob Chemother 50, 577-582.

Shlaes, D. M., Levy, J. \& Wolinsky, E. (1981). Enterococcal bacteremia without endocarditis. Arch Intern Med 141, 578-581.

Toledo-Arana, A., Valle, J., Solano, C. \& 7 other authors (2001). The Enterococcal Surface Protein, Esp, is involved in Enterococcus faecalis biofilm formation. Appl Environ Microbiol 67, 4538-4545. 